\title{
ISR and IFI in Precision AFB Studies with KKMC-hh
}

\author{
S.A. Yost ${ }^{* \dagger}$
}

The Citadel, The Military College of South Carolina

E-mail: scott.yost@citadel.edu

S. Jadach

Institute of Nuclear Physics IFJ-PAN, Krakow, Poland

E-mail: stanislaw.jadach@cern.ch

\section{B.F.L. Ward}

Baylor University

E-mail: bfl_ward@baylor.edu

\section{Z. Wąs}

Institute of Nuclear Physics, IFJ-PAN, Krakow, Poland

Email: wasmecern.ch

$\mathcal{K} \mathcal{K M C}$-hh is a hadronic event generator for $\mathrm{Z}$ boson production and decays, which includes exponentiated multi-photon radiation and first-order electroweak corrections. We have used $\mathcal{K} \mathcal{K} M C$ hh to investigate the role of initial sate radiation (ISR) and initial-final interference (IFI) in precision electroweak analyses at the LHC. We compare the effect of this radiation on angular distributions and forward-backward asymmetry, which are particularly important for the measurement of the weak mixing angle. We discuss the relation of the ISR implementation in $\mathcal{K} \mathcal{K} M C$-hh to ISR from parton distribution functions with QED corrections.

14th International Symposium on Radiative Corrections (RADCOR2019)

9-13 September 2019

Palais des Papes, Avignon, France

\footnotetext{
* Speaker.

$\dagger$ This presentation was supported by a grant from The Citadel Foundation. Computational resources were provided by the Institute of Nuclear Physics, IFJ-PAN, Krakow, Poland.

$\ddagger_{\text {This }}$ work was supported in part by Polish National Science Center grant 2016/23/B/ST2/03927.
} 


\section{Introduction}

Angular distributions for $p p \rightarrow Z / \gamma^{*} \rightarrow$ leptons are important for a precision measurement of the weak mixing angle at the LHC. The inputs for calculating the weak mixing angle can come from measurements of the forward-backward asymmetry $A_{\mathrm{FB}}$ or the angular coefficient $A_{4}=4\langle\cos \theta\rangle$. In either case, the relevant angle is taken to be the Collins-Soper (CS) angle in the rest frame of the final state lepton pair.[1]

The angular distribution is sensitive to radiative corrections. In the presence of final state radiation (FSR) from the leptons, the photon momenta can be subtracted to find the CM momentum of the $\mathrm{Z}$ boson. Initial state radiation (ISR) complicates this because it cannot be unambiguously distinguished from FSR. ISR also interferes with FSR at the quantum level, and this initial-final interference (IFI) creates an ambiguity in the $\mathrm{Z}$ boson rest frame that cannot be resolved, even in principle. These radiative effects are presently under investigation using a variety of programs in addition to $\mathcal{K K M C - h h , ~ i n c l u d i n g ~ P O W H E G - E W [ 2 ] ~ a n d ~ M C - S A N C [ 3 , ~ 4 ] . ~}$

We present studies of radiative corrections to angular distributions using $\mathcal{K} \mathcal{K M C}$-hh[5], a hadronic event generator based on CEEX[6], an amplitude-level soft photon exponentiation scheme originally developed for electron-positron collisions in the LEP era, which implemented for $e^{+} e^{-}$ scattering in the $\mathcal{K \mathcal { K M C }}$ generator[7] and extended to quark initial states in $\mathcal{K} \mathcal{K} M C$ 4.22[8]. CEEX is similar to YFS soft photon exponentiation[9], but implemented at the amplitude level rather than the cross section level, which facilitates the exponentiation of interference effects, in particular IFI. An extensive review and explanation of the implementation of IFI in the CEEX framework can be found in Ref. [10].

$\mathcal{K} \mathcal{K} M C$-hh events can be exported in an LHE-compatible [11] event record and showered by any external shower generator, or they can be showered by an internal implementation of HERWIG 6.5[12]. This assumes an approximation in which QCD and QED effects factorize, which is true at leading $\log$ and should be a good approximation at $\mathscr{O}\left(\alpha_{s} \alpha\right) \cdot[13,14]$ Unshowered events will be presented here, since the number of events needed to see the effect of radiative corrections on $A_{\mathrm{FB}}$ or $A_{4}$ is on the order of $10^{9}$ or more, requiring substantial computer resources, especially in the presence of the shower. A smaller sample of showered events was included in the RADCOR presentation[15], but was not discussed in detail and is not included here. It is expected that those results will be included in a more detailed analysis to be published soon.

$\mathcal{K} \mathcal{K} M C-h h$ includes an $a b$ initio calculation of QED radiation including quark masses, so that the results are finite in the collinear limit. This differs from other programs capable of addressing ISR effects in hadron scattering, such as POWHEG-EW, MC-SANC, Horace [16, 17], and ZGRAD2 [18], which factorize collinear QED radiation with the assumption that its effect is included in the parton distribution functions (PDFs). Factorizing the collinear QED has the advantage of avoiding the issue of quark masses, but setting a high factorization scale could limit the ability to address non-collinear ISR. Also, such factorization is not readily combined with CEEX soft photon exponentiation in $\mathcal{K} \mathcal{K M C}$-hh.

Including quark masses in the calculation raises the question of what value should be assigned to them. The first parton distributions to include QED corrections was the MRST QED PDF set[19], which assumed current quark masses. This is consistent with the expectation that for deep inelastic scattering, the colliding quarks couple perturbatively to the spectator quarks, so that the recoil 
when a photon is emitted should be governed by the current quark mass, not the constituent mass. However, some controversy remains on this issue, which was addressed in a study[20] applying $\mathcal{K} \mathcal{K M C}$-hh to LHC phenomenology relevant to the $W$ mass measurement by varying the quark masses. The mass dependence is logarithmic, so varying the light quark masses by a factor of 10 only changes the ISR contribution by about $10 \%$. Since ISR typically contributes at the order of around $0.1 \%$ for most distributions, the mass dependence is usually insignificant.

\section{The Effect of Initial-State QED Radiation on Angular Distributions}

In this section, we focus on CS angle distributions, particularly $A_{\mathrm{FB}}$ and $A_{4}$, and compare the effect of including QED corrections in the PDFs to the effect of adding ISR via $\mathcal{K} \mathcal{K M C}$-hh. All results are from $\mathcal{K} \mathcal{K} M C$-hh runs without a QCD shower, producing $5.7 \times 10^{9}$ muon events at 8 TeV. Since $\mathcal{K} \mathcal{K} M C$-hh includes collinear ISR, it must be used with pure-QCD parton distributions. These runs use NNPDF3.1[21] $\left(\alpha_{s}\left(M_{Z}\right)=0.21018\right)$. For comparison, we also show results for $\mathcal{K} \mathcal{K M C}$-hh with ISR off, but with a NNPDF3.1luxQED[22] parton distribution functions, which include LuxQED photon ISR[23].

NLO electroweak corrections are added using DIZET 6.21[24], which uses an input scheme with parameters $G_{\mu}, \alpha(0)$, and $M_{Z}$. The quark masses in DIZET are selected internally based on the vacuum polarization option, for which the default fit is used. Photonic radiative corrections are calculated using $\alpha(0)$ and PDG values[25] for the quark current masses. Otherwise, all parameters are consistent with the LHC electroweak benchmark study, Ref. [26].

All results include dilepton mass cut $60 \mathrm{GeV}<M_{l l}<116 \mathrm{GeV}$, including those labeled "uncut." The "cut" results include an additional constraint $p_{\mathrm{T}}>25 \mathrm{GeV}$ on the transverse momentum of each muon, and $|\eta|<2.5$ on the pseudorapidity of each muon. The forward-backward asymmetry $A_{\mathrm{FB}}$ is calculated from the cut events, while $A_{4}$ is calculated using uncut events. Final state radiation is included in all cases. Initial-final interference (IFI) is not included. IFI effects are discussed separately in the next section. In Table 1, the column labeled "No ISR" have ISR turned off in $\mathcal{K} \mathcal{K} M C$-hh and use a non-QED NNPDF3.1 set. The LuxQED column has ISR turned off

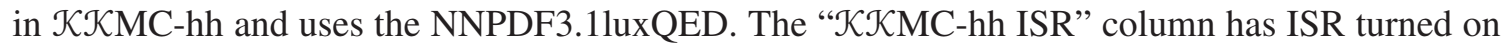
in $\mathcal{K} \mathcal{K} M C$-hh and uses a non-QED NNPDF3.1 set. Differences are shown comparing ISR on and off both ways, using LuxQED or $\mathcal{K} \mathcal{K} M C$-hh. In the case of the cross-section, the differences are shown as percentages, while for the asymmetries, the straight differences are shown.

\begin{tabular}{|l|c|c|c|c|c|}
\hline & No ISR & LuxQED ISR & LuxQED-no ISR & $\mathcal{K} \mathcal{K}$ MC-hh ISR & ISR-no ISR \\
\hline Uncut $\sigma$ & $939.858(7) \mathrm{pb}$ & $944.038(7) \mathrm{pb}$ & $0.445(1) \%$ & $944.99(2) \mathrm{pb}$ & $0.546(2) \%$ \\
Cut $\sigma$ & $439.103(7) \mathrm{pb}$ & $440.926(7) \mathrm{pb}$ & $0.415(1) \%$ & $442.36(1) \mathrm{pb}$ & $0.742(3) \%$ \\
$A_{\mathrm{FB}}$ & $0.01125(3)$ & $0.01145(2)$ & $(1.9 \pm 0.3) \times 10^{-4}$ & $0.1129(2)$ & $(3.9 \pm 2.8) \times 10^{-5}$ \\
$A_{4}$ & $0.06102(4)$ & $0.06131(3)$ & $(2.9 \pm 0.5) \times 10^{-4}$ & $0.06057(3)$ & $(-4.4 \pm 0.5) \times 10^{-4}$ \\
\hline
\end{tabular}

Table 1. Effect of ISR added via LuxQED or $\mathcal{K} \mathcal{K M C}$-hh

Both LuxQED and $\mathcal{K} \mathcal{K} M C$-hh show that ISR shifts the cut and uncut cross-section by about half a percent, with differences on the order of a per-mil. LuxQED also shows a shift in $A_{\mathrm{FB}}$ and 
$A_{4}$ on the order of a few per-mil, but the ISR effect in $\mathcal{K} \mathcal{K} M C$-hh is much smaller for $A_{\mathrm{FB}}$, and has the opposite sign for $A_{4}$.

Figures 1 and 2 compare Collins-Soper angular distributions $\cos \left(\theta_{\mathrm{CS}}\right)$ in three cases: "FSR only" has no ISR and a non- QED PDF set, "FSR + ISR" includes $\mathcal{K} \mathcal{K} M C$-hh ISR with a nonQED PDF set, and "FSR + LuxQED" uses a LuxQED PDF set with no ISR from $\mathcal{K} \mathcal{K} M C$-hh. Fig. 1 does not include the additional lepton cuts, and is the distribution relevant to $A_{4}$, while Fig. 2 includes the lepton cuts, and is relevant to $A_{\mathrm{FB}}$.

$\operatorname{Cos}\left(\theta_{c S}\right)$ Distribution: Without Cuts

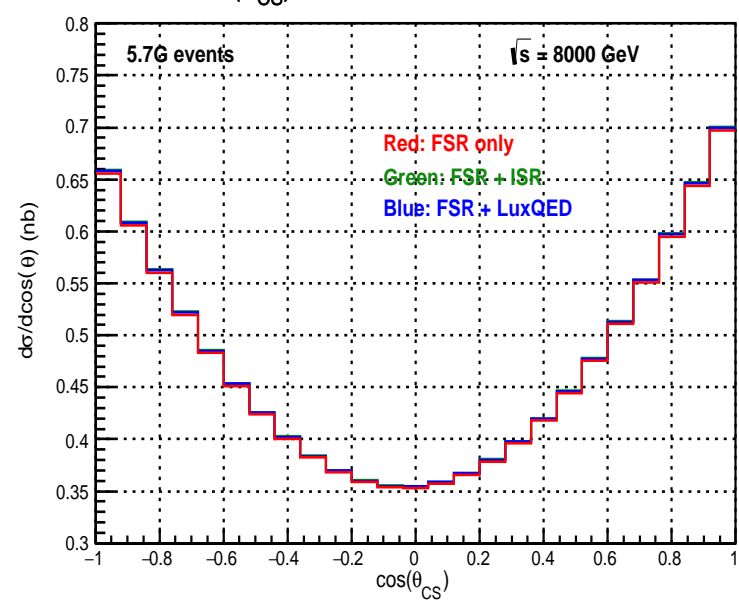

Fractional Contribution to Distribution

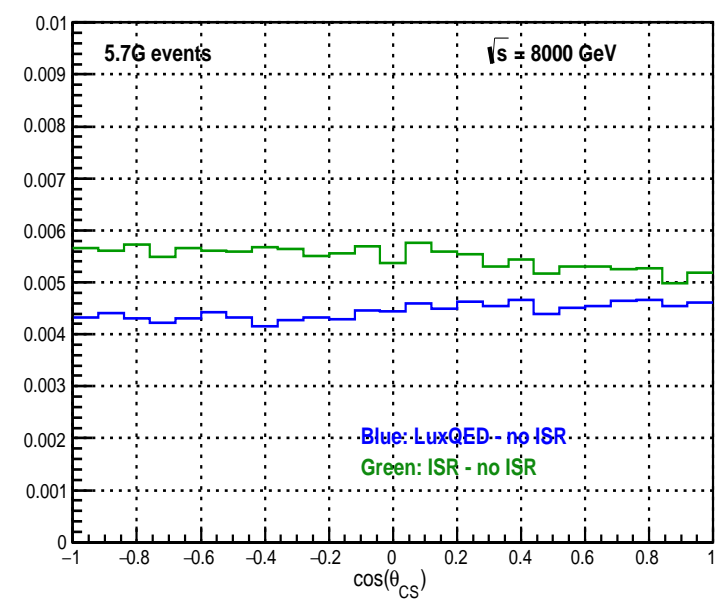

Figure 1: ISR contributions to $\cos \left(\theta_{\mathrm{CS}}\right)$ distributions, without lepton cuts.
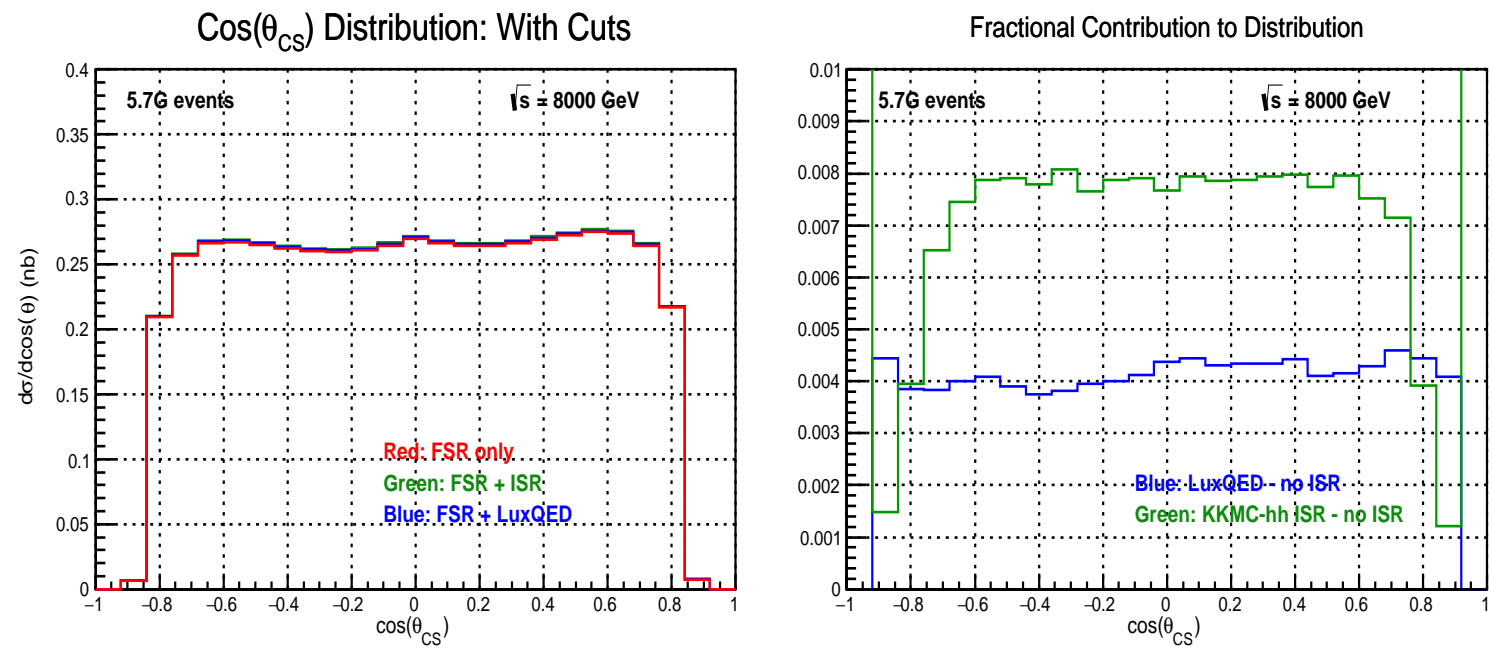

Figure 2: ISR contributions to $\cos \left(\theta_{\mathrm{CS}}\right)$ distributions, with lepton cuts.

Figures 3 and 4 show the effect of ISR on $A_{\mathrm{FB}}$ as a function of the dilepton mass and rapidity, respectively. In Fig. 3, the ISR contribution to $A_{\mathrm{FB}}$ is less than $10^{-3}$ for the entire range of $M_{l l}$, and in the vicinity of $M_{Z} \approx 91 \mathrm{GeV}$, it is less than $3 \times 10^{-4}$, for both LuxQED and $\mathcal{K} \mathcal{K} M C$-hh. In Fig. 
4, the ISR effect from $\mathcal{K} \mathcal{K M C}$-hh is below $10^{-4}$ in all bins, and consistent with zero in the central bin. However, LuxQED would give a larger ISR contribution for $Y_{l l}<2$.

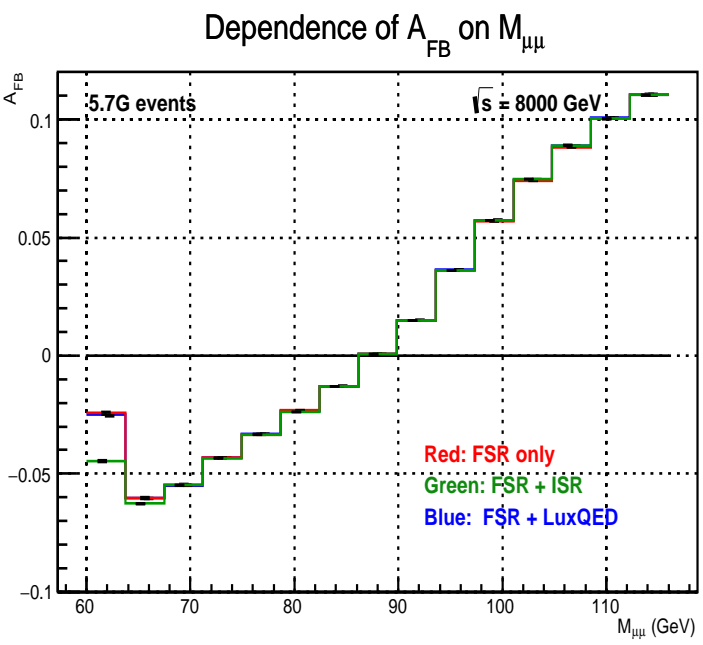

ISR contributions to $A_{F B}$

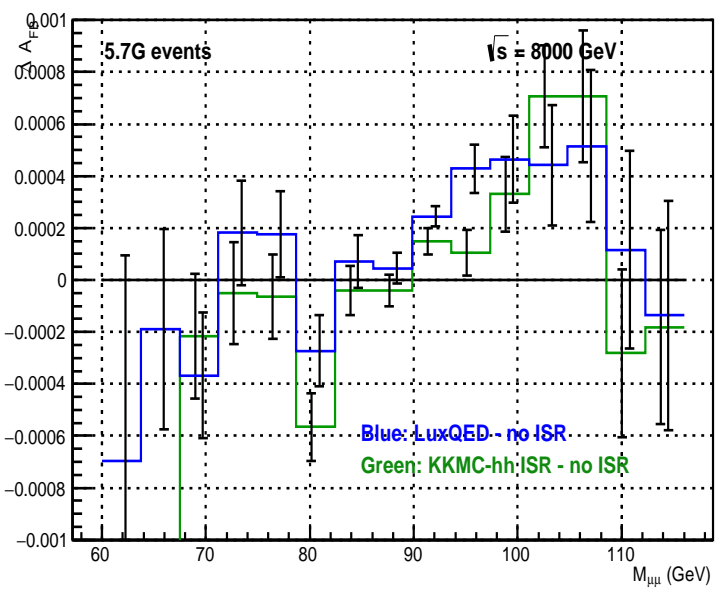

Figure 3: Effect of ISR on $A_{\mathrm{FB}}$ in terms of dilepton mass.
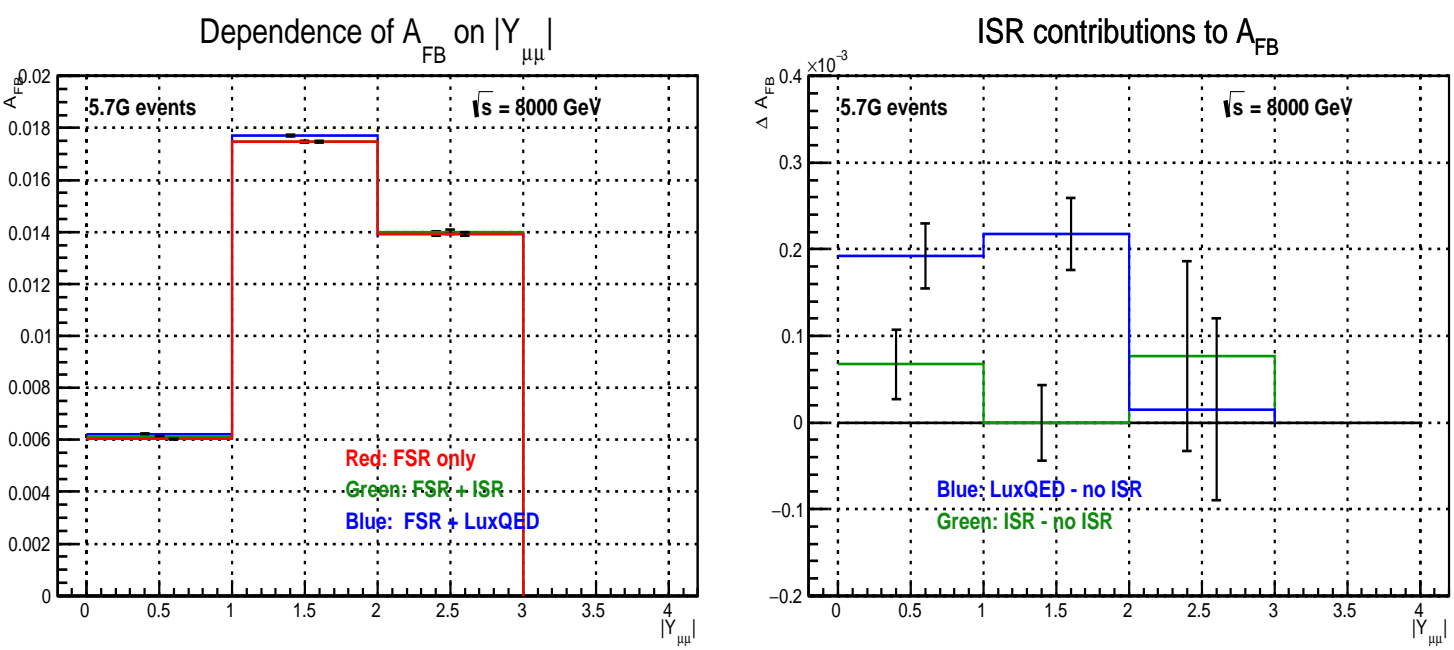

Figure 4: Effect of ISR on $A_{\mathrm{FB}}$ in terms of dilepton rapidity.

Figures 5 and 6 show the effect of ISR on $A_{4}$ as a function of the dilepton mass and rapidity.

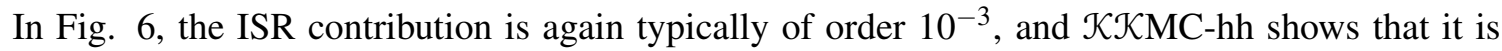
approximately consistent with zero in the vicinity of $M_{Z}$. In Fig. 7, the ISR contribution from $\mathcal{K \mathcal { K M C }}$-hh increases for large rapidity, but is on the order of $10^{-4}$ for $Y_{l l}<2$. The LuxQED prediction is consistently below $5 \times 10^{-4}$, but significantly different from $\mathcal{K} \mathcal{K} \mathrm{MC}$-hh.

\section{The Effect of Initial-Final Interference on Angular Distributions}

In this section, we consider the effect of quantum interference between initial and final state QED radiation (IFI) on the CS angular distributions, forward-backward asymmetry, and $A_{4}$. The 

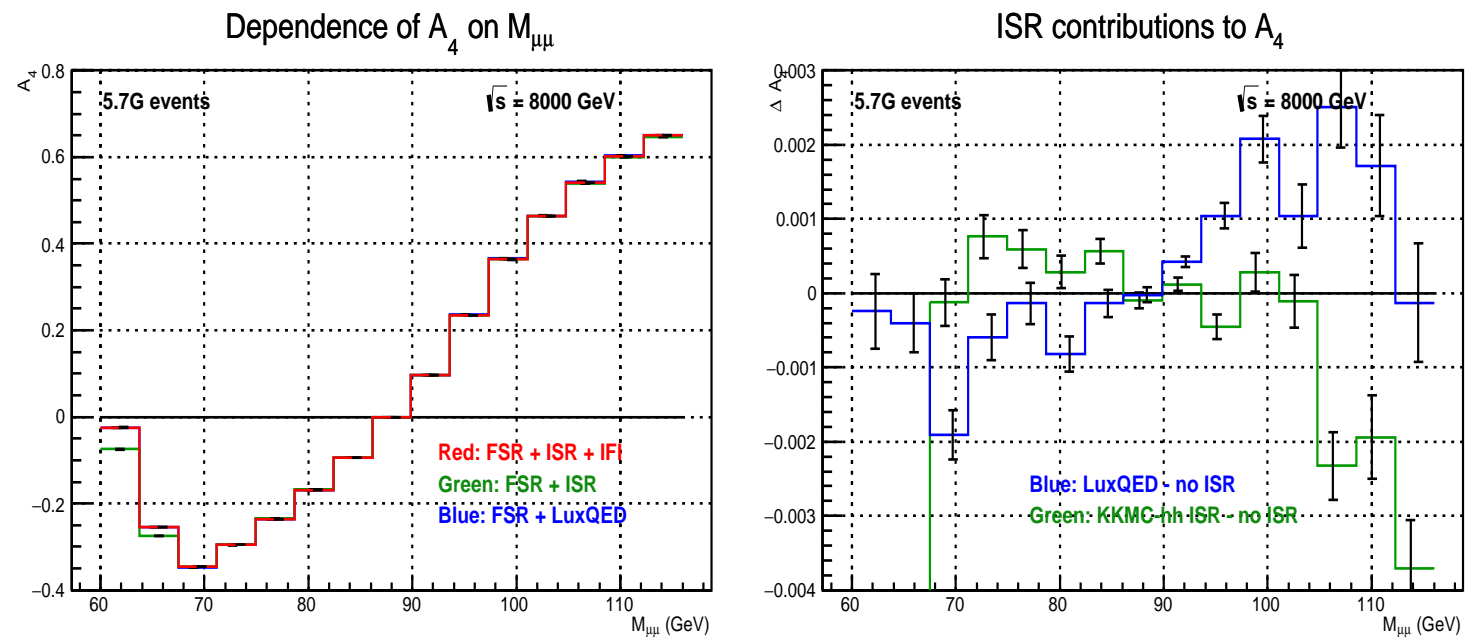

Figure 5: Effect of ISR on $A_{4}$ in terms of dilepton mass.

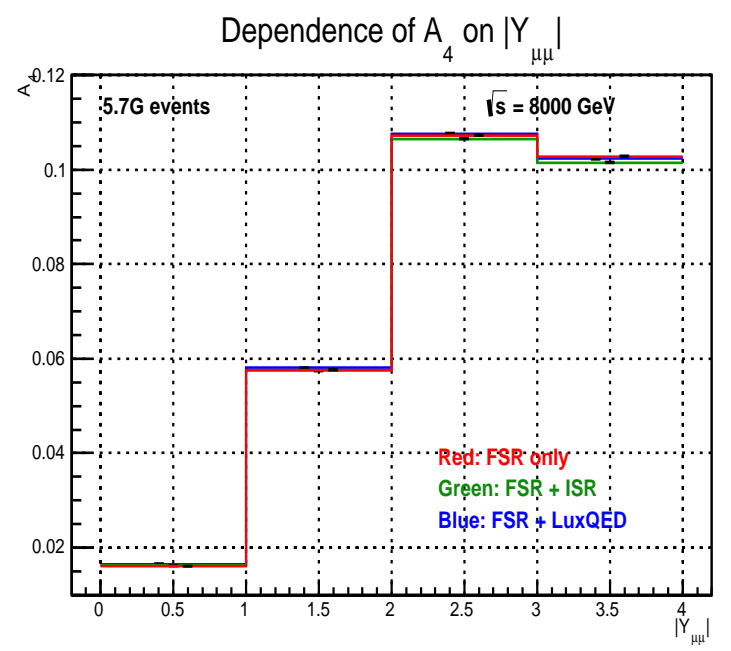

ISR contributions to $\mathrm{A}_{4}$

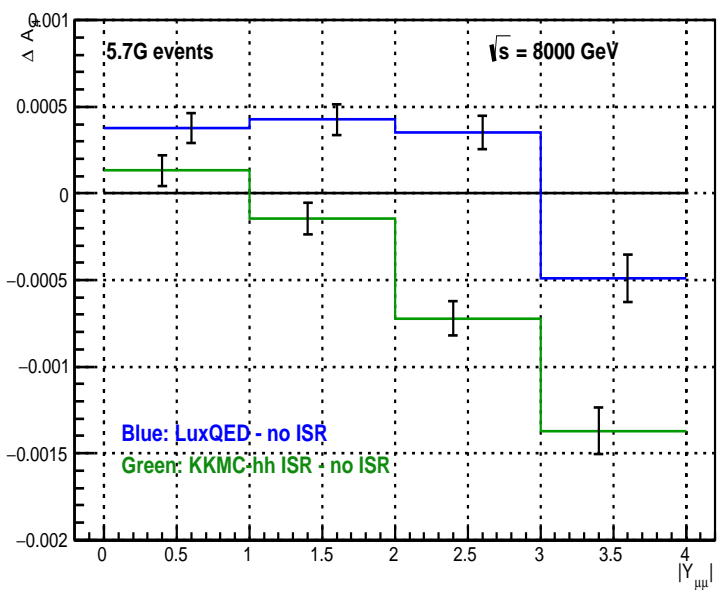

Figure 6: Effect of ISR on $A_{4}$ in terms of dilepton rapidity.

use of $A_{\mathrm{FB}}$ or $A_{4}$ in determining the weak mixing angle is complicated by IFI, it a quantum uncertainty in any attempt to back out FSR from the measurement.

All comparisons are without a QCD shower and use NNPDF3.1 parton distributions without QED corrections, since these are included in $\mathcal{K} \mathcal{K} M C$-hh. The parameters are the same as in the previous section.

Table 2 shows the effect of ISR on the uncut and cut cross sections as well as on the forward-

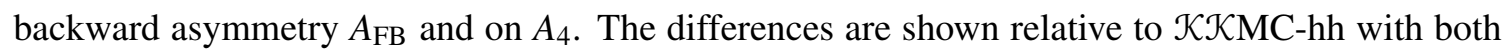
ISR and ISR on, but IFI off. For the cross sections, percent differences are shown, while for the asymmetries, the differences are shown directly. The comparisons were calculated within a single run by reweighting, and the errors take into account the weight correlations, which reduce the uncertainty. 


\begin{tabular}{|l|c|c|c|}
\hline & without IFI & with IFI & difference \\
\hline uncut $\sigma$ & $944.99(2)$ & $944.91(2)$ & $-0.0089(4) \%$ \\
cut $\sigma$ & $442.36(1)$ & $442.33(1)$ & $-0.0070(5) \%$ \\
$A_{\mathrm{FB}}$ & $0.01129(2)$ & $0.01132(2)$ & $(2.9 \pm 1.1) \times 10^{-5}$ \\
$A_{4}$ & $0.06057(3)$ & 0.061024 & $(4.5 \pm 0.3) \times 10^{-4}$ \\
\hline
\end{tabular}

Table 2. Effect of Initial-Final Interference

The contribution of IFI on cross sections is very small, of the order $0.01 \%$, while the effect of IFI on $A_{\mathrm{FB}}$ and $A_{4}$ is of order $10^{-5}$ and $10^{-4}$, respectively. The IFI-dependence of the uncut and cut CS angle distributions are shown in Fig. 7. The effect is typically a fraction of a per-mil, and angle-dependent. The uncut distribution (left) is relevant to $A_{4}$, and the cut distribution (right) is relevant to $A_{\mathrm{FB}}$.
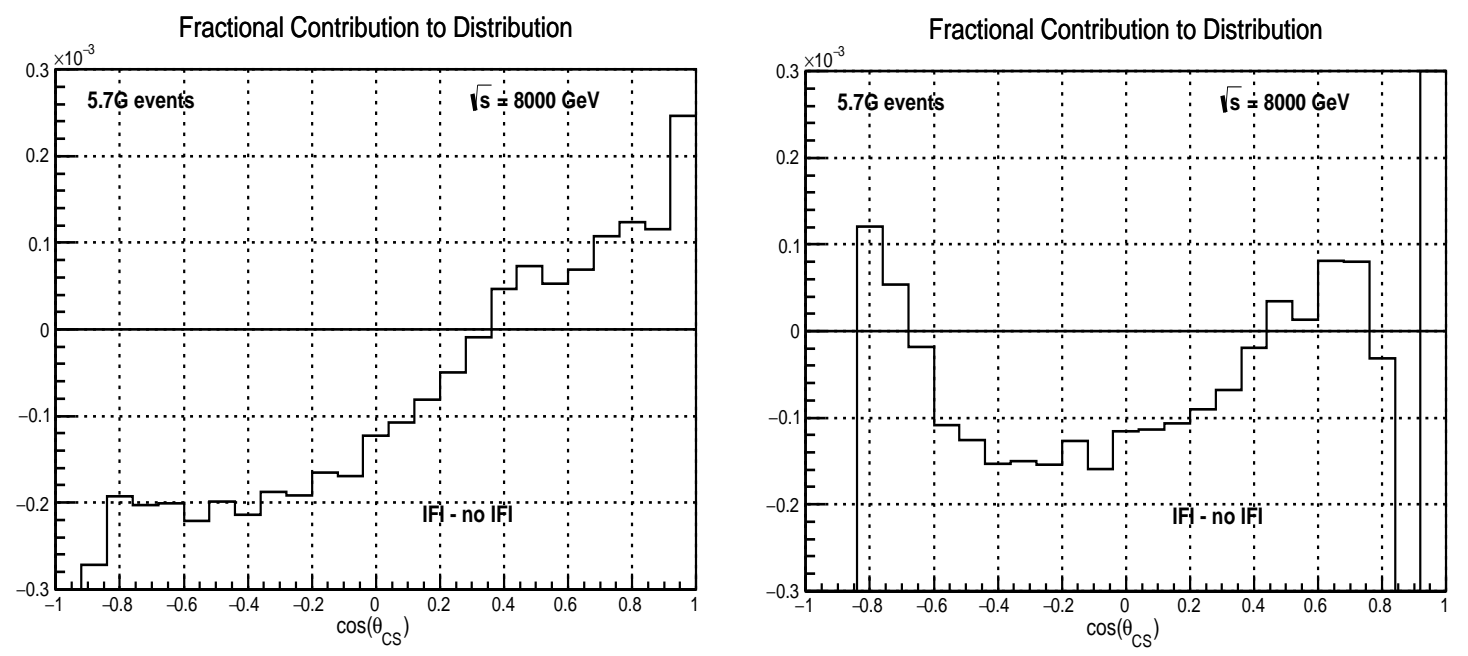

Figure 7: Dependence of the Collins-Soper angular distribution on initial-final interference, without lepton cuts (left) and with them (right).

Fig. 8 shows the IFI effect on the forward-backward asymmetry, as a function of the dilepton mass on the left, and the dilepton rapidity on the right. Fig. 9 shows similar comparisons for $A_{4}$. The IFI contribution to both $A_{\mathrm{FB}}$ and $A_{4}$ is consistent with zero near $M_{Z}$ and at low dilepton rapidity. However, $A_{4}$ is more sensitive to IFI than $A_{\mathrm{FB}}$ in general.

\section{Conclusions}

$\mathcal{K} \mathcal{K} M C$-hh provides a precise tool for calculating exponentiated photonic corrections to hadron scattering. We have presented estimates for the contributions of ISR and IFI to the $A_{\mathrm{FB}}$ and $A_{4}$ angular distributions which will be useful for determining the weak mixing angle from LHC data. $\mathcal{K K}$ MC-hh is particularly well suited to evaluating IFI due to its CEEX exponentiation, which was developed in part to facilitate the calculation of interference effects. The ISR contribution is large enough that it cannot be neglected in precision studies, and needs to be incorporated in some 
IFI contribution to $A_{F B}$

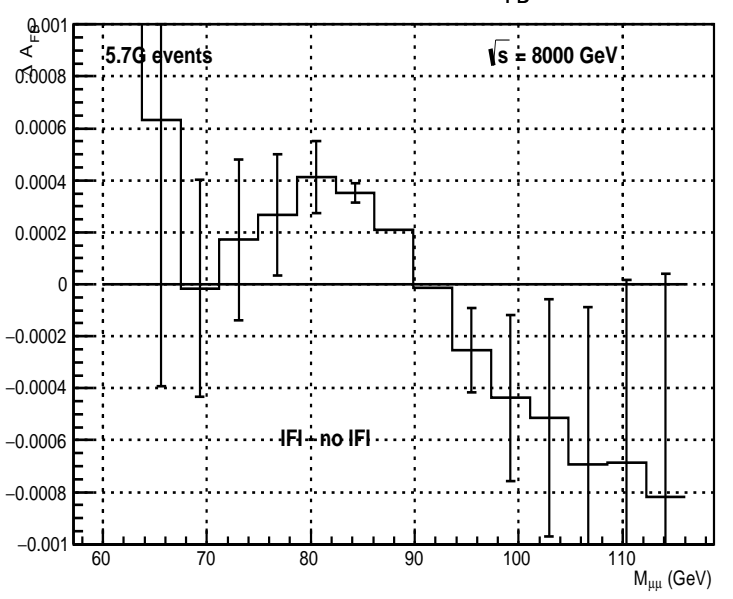

IFI contribution to $A_{F B}$

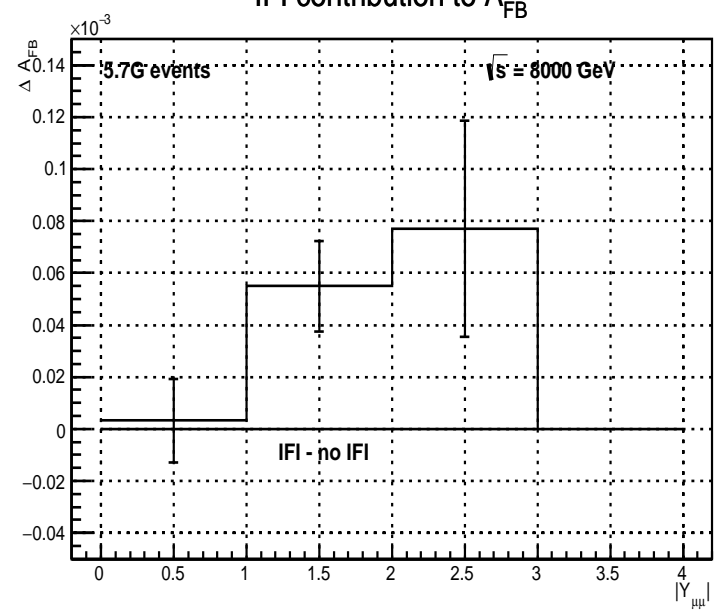

Figure 8: The IFI contribution to $A_{\mathrm{FB}}$ as a function of $M_{l l}$ (left) and $Y_{l l}$ (right).

IFI contribution to $\mathrm{A}_{4}$

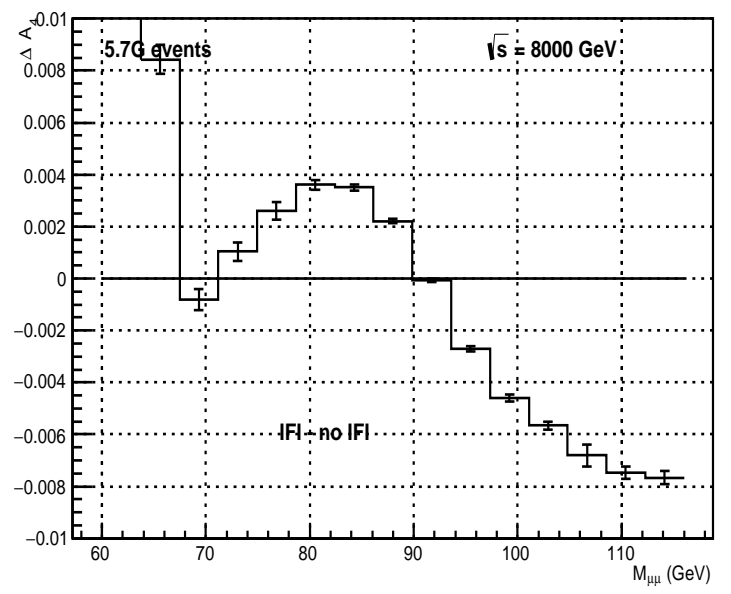

IFI contribution to $\mathrm{A}_{4}$

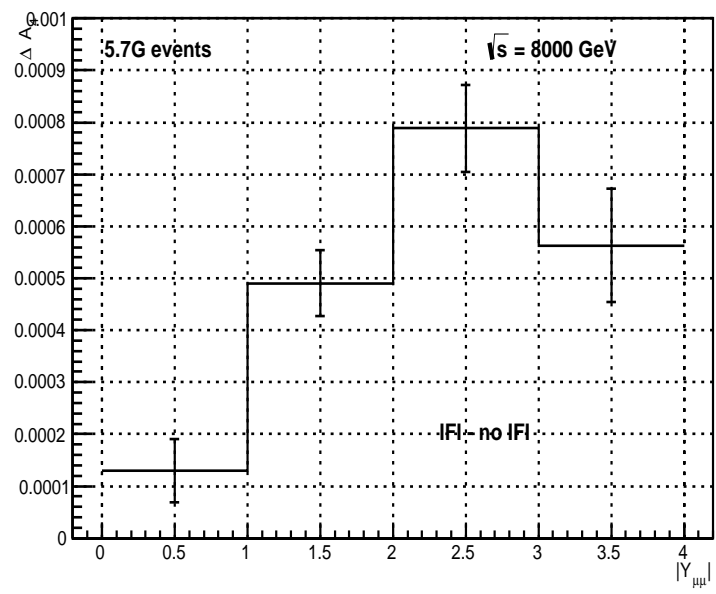

Figure 9: The IFI contribution to $A_{4}$ as a function of $M_{l l}$ (left) and $Y_{l l}$ (right).

manner, at least by including collinear photon emission in the PDFs, and preferably by including exponentiated photon emission in the generator, as in $\mathcal{K} \mathcal{K M C}$-hh.

The ab initio calculation of QED emission from the quarks is unique to the approach of $\mathcal{K K M M C - h h : ~ o t h e r ~ g e n e r a t o r s ~ u s e ~ c a l c u l a t i o n s ~ m a t c h e d ~ t o ~ a ~ Q E D - c o r r e c t e d ~ P D F ~ s e t . ~ S t u d i e s ~ c o m - ~}$ paring these approaches are in progress, and the results will be interesting not just at the computational level, but also conceptually, for better understanding the role of QED emission in hadron scattering.

Finally, we note that $\mathcal{K} \mathcal{K M C}$-hh is still under development. In the near future, we expect to be able to address NLO QCD issues as well, at first by adding a capability to add photonic corrections to events provided by any event generator, with the ansatz that QCD and QED corrections continue to factorize to an adequate approximation. Eventually, we anticipate incorporating NLO QCD internally, perhaps via the KrkNLO scheme.[27] 


\section{References}

[1] J.C. Collins and D.E. Soper, Phys. Rev. D16 (1977) 2219.

[2] L. Barzé, G. Montagna, P. Nason, et al., Eur. Phys. J. C73 (2013) 2474 [arXiv:1302.4606].

[3] S.G. Bondarenko and A.A. Sapronov, Comput. Phys. Commun. 184 (2013) 2343 [arXiv:1301.3687].

[4] A. Arbuzov, D. Bardin, S. Bondarenko, et al., JETP Lett. 103 (2016) no. 2, 131 [arXiv:1509.03052].

[5] S. Jadach, B.F.L. Ward, Z. Wạs, and S.A. Yost, Phys. Rev. D94 (2016) 074006 [arXiv:1608.01260].

[6] S. Jadach, B.F.L. Ward, and Z. Wa̧s, Phys. Rev. D63 (2001) 113009 [hep-ph/0006359].

[7] S. Jadach, B.F.L. Ward, and Z. Wa̧s, Comput. Phys. Commun. 130 (2000) 260 [hep-ph/9912214].

[8] S. Jadach, B.F.L. Ward, and Z. Wa̧s, Phys. Rev. D88 (2013) 114022 [arXiv:1307.4037].

[9] D.R. Yennie, S.C. Frautschi and H. Suura, Ann. Phys. 13 (1961) 379; K.T. Mahanthappa, Phys. Rev. 126 (1962) 329.

[10] S. Jadach and S.A. Yost, Phys. Rev. D100 (2019) 013002 [arXiv:1801.08611].

[11] E. Boos, M. Dobbs, W. Giele, et al., Generic User Process Interface for Event Generators, (2001) [hep-ph/0109068].

[12] G. Corcella, I.G. Knowles, G. Marchesini, et al., JHEP 0101 (2001) 010 [hep-ph/0011363].

[13] A. Denner, S. Dittmaier, T. Kasprzik and A. Mück, Eur. Phys. J. C73 (2013) 2297 [arXiv:1211.5078].

[14] S. Dittmaier, A. Huss, and C. Schwinn, Nucl. Phys. $B 855$ (2014) 318 [arXiv:1403.3216]; ibid., Nucl. Phys. B904 (2016) 216 [arXiv:1511.08016].

[15] S. Yost, ISR and IFI in Precision AFB Studies with $\mathcal{K} \mathcal{K} M C$-hh, RADCOR 2019, 12 Sept. 2019, indico.cern.ch/event/783212/contributions/3520688/.

[16] C.M. Carloni Calame, G. Montagna, O. Nicrosini and M. Treccani, Phys. Rev. D69 (2004) 037301; ibid., JHEP 0505 (2005) 019.

[17] C.M. Carloni Calame, G. Montagna, O. Nicrosini and A. Vicini, JHEP 0612 (2006) 016; ibid., JHEP 0710 (2007) 109.

[18] U. Baur, O. Brein, W. Hollik, et al., Phys. Rev. D65 (2002) 033007 [arXiv:hep-ph/0108274].

[19] A.D. Martin, W.J. Stirling, R.S. Thorne and G. Watt, Eur. Phys. J. C63 (2009) 189 [arXiv:0901.0002].

[20] S. Jadach, Z. Wa̧s, B.F.L. Ward and S.A. Yost, Phys. Rev. D99 (2019) 076016. [arXiv.1707.06502].

[21] R.D. Ball, Bertone, V., Carrazza, N.P, et al., Eur. Phys. J. C77 (2017) 77:663 [arXiv:1706.00428].

[22] V. Bertone, S. Carrazza, N.P. Hartland and J. Rojo, SciPost Phys. 5 (2018) 008 [arXiv:1712.07053].

[23] A. Manohar, P. Nason, G.P. Salam and G. Zanderighi, Phys. Rev. Lett. 117 (2016) 242002 [arXiv:1607.04266]; ibid., JHEP 12 (2017) 046 [arXiv:1708.01256].

[24] D. Bardin, M. Bilenky, P. Christova, et al., Comput. Phys. Commun. 133 (2001) 229 [hep-ph/9908433].

[25] Particle Data Group, Phys. Rev. D98 (2018) 030001.

[26] S. Alioli, A.B. Arbuzov, D.Yu. Bardin, et al., Eur. Phys. J. C77 (2017) 280 [arXiv:1606.02330].

[27] S. Jadach, A. Kusina, W. Płaczek, et al., Phys. Rev. D87 034029 [arXiv:1103.5015]; ibid., Acta Phys. Polon. B46 (2015) 2089; ibid., JHEP 10 (2015) 052 [arXiv:1503.06849]. 